\title{
Ising Model in the High Density Limit
}

\author{
Colin J. Thompson \\ Mathematics Department, University of Melbourne, Victoria, Australia \\ Received January 6, 1974
}

\begin{abstract}
It is proved that the free energy per spin in the thermodynamic limit of an Ising model on a lattice with coordination number $z$ approaches the classical Curie-Weiss free energy in the limit $z \rightarrow \infty$. The infinite spacial dimension limit of nearest neighbour lattice models is a special case of this result.
\end{abstract}

\section{Introduction}

It is well known that in a variety of circumstances one obtains the classical theories of phase transitions for fluid and magnetic systems in the limit of long range interactions. Such results to date (e.g. Lebowitz and Penrose [1], Thompson and Silver [2]) have been concerned with attractive pair potentials

$$
\phi(\boldsymbol{r})=\gamma^{d} v(\gamma|\boldsymbol{r}|)
$$

of Kac type, in fixed spacial dimension $d$, in the limit $\gamma \rightarrow 0$.

Various expansions for thermodynamic quantities in powers of $\gamma$ have been obtained $[3,4]$, and resummed in regions close to the critical point [5]. Prior to Refs. [1-5], Brout [6] obtained high density expansions for spin systems on lattices in inverse powers of the coordination number $z$ of the lattice, with the classical Curie-Weiss theory as leading term. On the basis of Brout's work it has often been stated (e.g. [7]) that critical behaviour for spin systems on $d$-dimensional lattices $(z=2 d$ for a regular cubic lattice) should become classical in the limit $d \rightarrow \infty$.

Our purpose here is to prove that the $z \rightarrow \infty$ limit for an Ising model on a lattice with coordination number $z$ indeed results in the classical Curie-Weiss theory. We stress that the lattice does not have to be regular or tied to a particular spacial dimension. All that is required is that each of the $N$ points of the lattice be bonded or linked to $z$ other points of the lattice.

The precise statement is as follows.

Theorem. Let each point $p$ of a lattice with $N$ points and coordination number $z$ be occupied by a spin $\mu_{p}= \pm 1$ and let the spins interact with 
energy

$$
E\{\mu\}=-\frac{J}{z} \sum_{p, p^{\prime}}^{*} \mu_{p} \mu_{p^{\prime}}-H \sum_{p} \mu_{p}
$$

in a given configuration $\{\mu\}$, where $J \geqq 0$ and $H$ are constants and the starred sum is over the $N z$ pairs $\left(p, p^{\prime}\right)$ of linked points.

Then the free energy per spin $\psi_{z}(\beta, H)$ defined by $\left(\beta=(k T)^{-1}\right)$

with

$$
-\beta \psi_{z}(\beta, H)=\lim _{N \rightarrow \infty} N^{-1} \log Z_{N}(\beta, H)
$$

has the limiting form

$$
Z_{N}(\beta, H)=\sum_{\{\mu\}} \exp (-\beta E\{\mu\})
$$

$$
\lim _{z \rightarrow \infty}\left[-\beta \psi_{z}(\beta, H)\right]=\operatorname{Max}_{m}\left[-v m^{2}+\log 2 \cosh (2 v m+B)\right]
$$

where $B=\beta H$ and $v=\beta J$.

It will be noted that (1.5) corresponds to the classical Curie-Weiss theory of magnetism. It should also be noted that the factor $1 / z$ in (1.2) is needed for the limit (1.5) to exist.

Although dimensionality does not appear explicitly in the statement of the theorem we have in mind, as a particular case, the nearest neighbour Ising model on a $d$-dimensional cubic lattice (for which $z=2 d$ ). The limit $z \rightarrow \infty$ then corresponds to infinite spacial dimensionality.

We have nothing to say here about the validity of classical critical behaviour in (fixed) finite integer dimensions larger than three (see [8] and [9]). In any event it is probably correct to say that the free energy will have the Curie-Weiss form only in the limit $z \rightarrow \infty$ (or $d \rightarrow \infty)$. This of course does not exclude the possibility of classical critical exponents for finite $d \geqq 4$.

\section{Proof of the Theorem}

In the proof of the Theorem we adopt the usual strategy of bounding the partition function $Z_{N}$ (1.4) above and below and showing that the two corresponding bounds for the limiting free energy per spin coalesce in the limit $z \rightarrow \infty$.

As shown in [2], the Curie-Weiss partition function is always a lower bound for $Z_{N}$, regardless of potential or dimension. In the present context, elementary manipulation leads to the identity

$$
\begin{aligned}
Z_{N}(\beta, H) & =\sum_{\{\mu\}} \exp \left(\frac{v}{z} \sum_{p, p^{\prime}}^{*} \mu_{p} \mu_{p^{\prime}}+B \sum_{p} \mu_{p}\right) \\
& =\left[e^{-v m^{2}} 2 \cosh (2 v m+B)\right]^{N}\left\langle\exp \left(\frac{v}{z} \sum_{p, p^{\prime}}^{*} \sigma_{p} \sigma_{p^{\prime}}\right)\right\rangle_{c}
\end{aligned}
$$


where $m$ is any constant,

$$
\sigma_{p}=\mu_{p}-m
$$

and the average $\langle\cdots\rangle_{c}$ is taken with respect to the distribution function

$$
P\{\mu\}=\exp \left[(2 v m+B) \sum_{p} \mu_{p}\right] /[2 \cosh (2 v m+B)]^{N} .
$$

In deriving (2.1), which is an identity for all $m$, we have used the facts that $\sum_{p}^{*}=z$ and that as a consequence $\sum_{p, p^{\prime}}^{*}=z N$.

Using Jensen's inequality $(\langle\exp \chi\rangle \geqq \exp \langle\chi\rangle)$ we find from (2.1) that

$$
\begin{aligned}
Z_{N}(\beta, H) & \geqq\left[e^{-v m^{2}} 2 \cosh (2 v m+B)\right]^{N} \exp \left(\frac{v}{z} \sum_{p, p^{\prime}}^{*}\left\langle\sigma_{p}\right\rangle_{c}\left\langle\sigma_{p^{\prime}}\right\rangle_{c}\right) \\
& =\left[e^{-v m^{2}} 2 \cosh (2 v m+B)\right]^{N},
\end{aligned}
$$

provided $m$ is chosen such that

$$
\left\langle\sigma_{p}\right\rangle_{c}=0
$$

i.e. from (2.2) and (2.3)

$$
\left\langle\mu_{p}-m\right\rangle=\tanh (2 v m+B)-m=0 .
$$

It follows that

$$
\begin{aligned}
-\beta \psi_{z}(\beta, H) & =\lim _{N \rightarrow \infty} N^{-1} \log Z_{N}(\beta, H) \\
& \geqq-v m^{2}+\log 2 \cosh (2 v m+B)
\end{aligned}
$$

where from (2.6) $m$ can be chosen as the solution that maximises the right hand side of (2.7).

It remains to show that the right hand side becomes an upper bound for $-\beta \psi_{z}(\beta, H)$ in the limit $z \rightarrow \infty$.

To obtain an upper bound on $Z_{N}(\beta, H)$ we begin by expanding the exponential in (2.1) obtaining

where

$$
Z_{N}(\beta, H)=\left[e^{-v m^{2}} 2 \cosh (2 v m+B)\right]^{N} I(N, z)
$$

$$
\begin{gathered}
I(N, z)=\sum_{k=0}^{\infty}\left(\frac{2 v}{z}\right)^{k} \frac{1}{k !} I_{k}, \\
I_{k}=2^{-k} \sum_{P_{1}, \ldots, P_{k}}^{*}\left\langle\left(\sigma_{p_{1}{ }^{\prime}} \sigma_{p_{1^{\prime}}{ }^{\prime \prime}}\right)\left(\sigma_{p_{2^{\prime}}} \sigma_{p_{2^{\prime}}}\right) \ldots\left(\sigma_{p_{k^{\prime}}} \sigma_{p_{k^{\prime}}{ }^{\prime \prime}}\right)\right\rangle_{c},
\end{gathered}
$$

and the sum in (2.10) is over linked pairs of points $P_{i}=\left(p_{i}^{\prime}, p_{i}^{\prime \prime}\right), i=1, \ldots, k$.

The quantity $I_{k}$ can be expressed in terms of weighted labelled graphs as follows. 
To each factor $\sigma_{p^{\prime}} \sigma_{p^{\prime \prime}}$ in (2.10) we associate a bond on the lattice connecting the points $p^{\prime}$ and $p^{\prime \prime}$. With such an association, every term in the sum (2.10) can be represented by a graph on the lattice consisting of $k$ bonds. The sum (2.10) then becomes a sum of weighted labelled graphs. Thus given a graph $G_{k}$ of $k$ labelled bonds $P_{1}, P_{2}, \ldots, P_{k}$ $\left(P_{i}\right.$ connecting points $\left.p_{i}^{\prime}, p_{i}^{\prime \prime}\right)$ we denote the corresponding weight $\left\langle\left(\sigma_{p_{1}{ }^{\prime}} \sigma_{p_{1^{\prime \prime}}}\right) \ldots\left(\sigma_{p_{k^{\prime}}} \sigma_{p_{k^{\prime \prime}}}\right)\right\rangle$ by $W\left(G_{k}\right)$. If we then restrict the sum over bonds so that each bond is counted only once, the factor $2^{k}$ in (2.10) is eliminated and we can write

$$
I_{k}=\sum_{\left(G_{k}\right)} W\left(G_{k}\right)
$$

Now, since $W\left(G_{k}\right)$ is independent of the labelling of $G_{k}$ and is a product of weights assigned to each of the disjoint connected parts making up $G_{k}$ we can write $I(N, z)$ Eq. (2.9), as (see for example [10])

where

$$
I(N, z)=\exp [i(N, z)]
$$

$$
i(N, z)=\sum_{k=1}^{\infty}\left(\frac{2 v}{z}\right)^{k} \frac{i_{k}}{k !}
$$

and

$$
i_{k}=\sum_{\left(C_{k}\right)} W\left(C_{k}\right)
$$

the sum in (2.14) now being over connected labelled graphs $C_{k}$ of $k$ bonds.

To complete the proof of our theorem we need the following:

Lemma. $i_{k}$ defined by (2.14) satisfies the inequality

$$
\left|i_{k}\right| \leqq \frac{N k !}{z}\left(\frac{A z}{2 v}\right)^{k}, \quad A<1
$$

for all fixed $v>0$ and sufficiently large $z$.

The proof of the lemma is given in the appendix.

Substituting (2.15) in (2.12) and (2.13) we obtain

$$
I(N, z) \leqq \exp \left(\frac{N A}{z(1-A)}\right)
$$

for $z$ sufficiently large.

It follows then from (2.8) that

$$
-\beta \psi_{z}(\beta, H) \leqq-v m^{2}+\log 2 \cosh (2 v m+B)+\frac{A}{z(1-A)}
$$

and hence from $(2.7)$ that $\psi_{z}(\beta, H)$ in the limit $z \rightarrow \infty$ is given by the Curie-Weiss free energy (1.5).

The proof of the theorem is then complete. 


\section{Appendix}

Our aim here is to prove the result (2.15),

$$
\left|\sum_{\left(C_{k}\right)} W\left(C_{k}\right)\right| \leqq \frac{N k !}{z}\left(\frac{z A}{2 v}\right)^{k}, \quad A<1
$$

$v$ fixed, for $z$ sufficiently large.

To estimate the weight of a graph recall definitions (2.10) and (2.11) and the fact that spins occur independently in the distribution function (2.3). As a consequence a graph $G$ with $m_{f}$ vertices of degree $f$ has weight

$$
W(G)=\prod_{f \geqq 1}\left(\left\langle\sigma^{f}\right\rangle\right)^{m_{f}}
$$

where for example [from (2.6)]

$$
\begin{aligned}
\langle\sigma\rangle & =\langle\mu-m\rangle=0, \\
\left\langle\sigma^{2}\right\rangle & =\left\langle(\mu-m)^{2}\right\rangle \\
& =\left\langle\left(1-2 m \mu+m^{2}\right)\right\rangle \\
& =1-m^{2}
\end{aligned}
$$

and so on. The result (A 3), which is very important for our purposes, means that graphs containing points of degree one have zero weight and can therefore be discarded.

To obtain the required result (A 1) we need to consider vertices of degree two [and weight (A4)] separately from vertices of higher degree. For vertices of degree $f \geqq 3$ the following trivial bound

$$
\left|\left\langle(\mu-m)^{f}\right\rangle\right| \leqq(1+m)^{f}
$$

is sufficient for our needs. (It should be noted that $\left\langle\sigma^{f}\right\rangle \neq 0$ for odd $f \geqq 3$ unless $m=0$.)

Noting now that if a graph has $k$ bonds and $m_{f}$ points of degree $f \geqq 2$ (and none of degree one) $k$ is given by

Eqs. (A 2) to (A 5) give

$$
k=\frac{1}{2} \sum_{f \geqq 2} f m_{f}
$$

$$
\begin{aligned}
\left|W\left(C_{k}\right)\right| & \leqq\left(1-m^{2}\right)^{m_{2}}(1+m)^{\sum_{f \geqq 3} f m_{f}} \\
& =\left(1-m^{2}\right)^{m_{2}}(1+m)^{2\left(k-m_{2}\right)} \\
& =\left(1-m^{2}\right)^{k} x^{k-m_{2}}
\end{aligned}
$$

where

$$
x=(1+m)(1-m)^{-1} \text {. }
$$

It follows that

$$
\left|\sum_{\left(C_{k}\right)} W\left(C_{k}\right)\right| \leqq\left(1-m^{2}\right)^{k} \sum_{m_{2}=0}^{k} n_{k}\left(m_{2}\right) x^{k-m_{2}}
$$


where $n_{k}\left(m_{2}\right)$ is the number of labelled graphs of $k$ bonds with $m_{2}$ vertices of degree two (and none of degree one) that can be drawn on the lattice.

To get a feel for $n_{k}\left(m_{2}\right)$ let us consider the case $m_{2}=k$, i.e. all vertices are of degree two. Obviously $n_{k}(k)$ is then the number of (self avoiding) labelled cycles of $k$ bonds that can be drawn on the lattice. If we consider a cycle as a self avoiding walk beginning and ending at a particular point $P$ say, it is clear that

$$
n_{k}(k) \leqq N k ! s_{k}
$$

where $s_{k}$ is the number of (unlabelled) self avoiding closures on $P$, the $k$ ! is the number of ways of labelling the bonds or steps of the walk [since $P$ can be chosen in any one of $k$ ways, $k$ ! could be replaced in (A 10) by $(k-1) !]$ and the factor $N$ is the number of possible positions for $P$ on the lattice.

To bound $s_{k}$ we note that given any self avoiding closure on a point $P$ we can construct $z-1$ unique walks on the lattice by replacing the last step by a step in any one of the $z-1$ remaining directions. For every cycle therefore we have $z$ unique random walks (not necessarily self avoiding) and since there is a total of $z^{k}$ random walks of $k$ steps from $P$ on a lattice with coordination number $z$ we have

and hence from (A 10)

$$
z S_{k} \leqq z^{k}
$$

$$
n_{k}(k) \leqq N k ! z^{k-1}
$$

In the general case we represent graphs on the lattice containing $k$ bonds as pseudo-graphs of $l=k-m_{2}$ lines (corresponding to chains of bonds connecting vertices of degree two) and $p=\sum_{f \geqq 3} m_{f}$ points (corresponding to vertices of degree at least three). Lines will be assigned lengths $k_{1}, k_{2}, \ldots, k_{l}\left(\sum_{i=1}^{l} k_{i}=k\right)$ corresponding to the number of bonds making up the associated chains on the lattice.

Now from Euler's theorem, a graph with $l$ lines and $p$ points contains $c=l-p+1$ independent cycles, corresponding as before to self avoiding closures on the lattice. By breaking each cycle as above we can construct $z^{c}$ Cayley trees (i.e. pseudo-graphs with no cycles). Hence if $N_{k}\left\{m_{f}\right\}$ is the number of graphs of $k$ bonds and $m_{f}$ points of degree $f(\geqq 2)$ we obtain as a straightforward generalization of (A11) and (A 12),

$$
\begin{aligned}
z^{c} N_{k}\left\{m_{f}\right\} & \leqq N T_{k, l} \sum_{k_{1}+k_{2}+\cdots+k_{l}=k} z^{k_{1}} z^{k_{2}} \ldots z^{k_{l}} \\
& \leqq N z^{k} T_{k, l}\left(\begin{array}{l}
k \\
l
\end{array}\right)
\end{aligned}
$$


i.e. since $c=k-\sum_{f \geqq 2} m_{f}+1$,

$$
N_{k}\left\{m_{f}\right\} \leqq N z^{\sum^{j} 2^{2} m_{f}-1} T_{k, l}\left(\begin{array}{l}
k \\
l
\end{array}\right)
$$

where $T_{k, l}$ is the number of trees with $l$ lines and $k$ labelled "components" (corresponding to bonds on the lattice). Using the fact that there are $p^{p-2}$ trees with $p$ labelled points and noting that a tree with $p$ points has $p-1$ lines, we have

$$
T_{k, l} \leqq\left(\begin{array}{l}
k \\
l
\end{array}\right)(l+1)^{l-1}(k-l) !
$$

where the first factor is the number of ways of choosing $l$ labels out of $k$, the second factor is the number of $(l-)$ labelled trees and the third factor is the number of ways of labelling the $k-l$ remaining components.

Combining (A 13) and (A 14) and using the fact that $p^{p} / p ! \leqq e^{p-1}$ we then have

$$
N_{k}\left\{m_{f}\right\} \leqq N k ! e^{l}\left(\begin{array}{l}
k \\
l
\end{array}\right) z^{f \geqq 2} m_{f}-1
$$

and hence, since $l=k-m_{2}$,

$$
\begin{aligned}
n_{k}\left(m_{2}\right) & =\Sigma^{\prime} N_{k}\left\{m_{f}\right\} \\
& \leqq N k !\left(\begin{array}{c}
k \\
k-m_{2}
\end{array}\right) e^{k-m_{2}} \Sigma^{\prime} z^{j \geqq 2} m_{f}-1
\end{aligned}
$$

where the primed sum is over $m_{3}, \ldots, m_{2 k} \geqq 0$ such that

$$
\sum_{f \geqq 3} f m_{f}=2\left(k-m_{2}\right) .
$$

Making use of (A 17) we can write

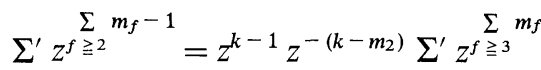

$$
\begin{aligned}
& =z^{k-1} \Sigma^{\prime} z^{-1 / 2 \sum_{f \geqq 3}(f-2) m_{f}} \text {. }
\end{aligned}
$$

Now since

$$
\begin{aligned}
1 / 2 \sum_{f \geqq 3}(f-2) m_{f} & =1 / 10 \sum_{f \geqq 3} f m_{f}+2 / 5 \sum_{f \geqq 3}(f-5 / 2) m_{f} \\
& \geqq 1 / 5\left(k-m_{2}+\sum_{f \geqq 3} m_{f}\right),
\end{aligned}
$$

we have

$$
\begin{aligned}
\Sigma^{\prime} z^{-1 / 2} \sum_{f \geqq 3}(f-2) m_{f} & \leqq z^{-\left(k-m_{2}\right) / 5} \sum_{m_{3}, \ldots, m_{2 k}=0}^{\infty} z^{-1 / 5 \sum_{f \geqq 3} m_{f}} \\
& \leqq z^{-\left(k-m_{2}\right) / 5}\left(1-z^{-1 / 5}\right)^{-2 k}
\end{aligned}
$$


Combining (A 16)-(A 19) we then have

$$
n_{k}\left(m_{2}\right) \leqq \frac{N k !}{z}\left[z\left(1-z^{-1 / 5}\right)^{-2}\right]^{k}\left(\begin{array}{c}
k \\
k-m_{2}
\end{array}\right)\left(\frac{e}{z^{1 / 5}}\right)^{k-m_{2}},
$$

hence from (A9)

$$
\left|\sum_{\left(C_{k}\right)} W\left(C_{k}\right)\right| \leqq \frac{N k !}{z}\left(z \frac{\left(1-m^{2}\right)\left(1+e x z^{-1 / 5}\right)}{\left(1-z^{-1 / 5}\right)^{2}}\right)^{k}
$$

which is (A 1) with $A$ given by

$$
A=2 v\left(1-m^{2}\right)\left(1+e x z^{-1 / 5}\right)\left(1-z^{-1 / 5}\right)^{-2} .
$$

Recalling that $m$ is the positive solution (assuming $H>0$ ) of

$$
m=\tanh (2 v m+B)
$$

and noting that the slope of the curve $y=\tanh (2 v m+B)$ is strictly less than unity where it intersects $y=m(>0)$, we have for all $v$ and $H>0$ that

$$
2 v\left(1-m^{2}\right)<1
$$

and hence from (A21) that $A<1$ for all $v$ and sufficiently large $z$.

The proof of (A 1) is then complete.

Acknowledgement. I would like to thank David J. Gates for a critical reading of the manuscript and for several helpful discussions.

\section{References}

1. Lebowitz,J.L., Penrose, O.: J. Math. Phys. 7, 98 (1966)

2. Thompson, C.J., Silver, H.: Commun. math. Phys. 33, 53 (1973)

3. Siegert, A.J.F., Vezzetti, D.J.: J. Math. Phys. 9, 2173 (1968)

4. Kac, M., Thompson, C. J.: J. Math. Phys. 10, 1373 (1969)

5. Thompson, C.J., Siegert, A.J.F., Vezzetti, D.J.: J. Math. Phys. 11, 1018 (1970)

6. Brout, R.: Phys. Rev. 118, 1009 (1960)

7. Fisher,M.E., Gaunt,D.S.: Phys. Rev. 133, A 224 (1964)

8. Wilson, K.G., Fisher,M.E.: Phys. Rev. Letters 28, 240 (1972)

9. Wilson, K. G.: Phys. Rev. Letters 28, 548 (1972)

10. Uhlenbeck, G.E., Ford, G. W.: Studies in statistical mechanics, Vol. 1, p. 131. Amsterdam: North Holland 1962

Communicated by G. Gallavotti

C. J. Thompson

University of Melbourne

Department of Mathematics

Parkville, Victoria 3052, Australia 